\title{
Dust in the eye of Andromeda
}

\author{
K. A. Marsh, ${ }^{\star}$ A. P. Whitworth, M. W. L. Smith, O. Lomax and S. A. Eales \\ School of Physics and Astronomy, Cardiff University, Cardiff CF24 3AA, Wales, UK
}

Accepted 2018 July 26. Received 2018 July 26; in original form 2018 April 12

\begin{abstract}
We present new Herschel-derived images of warm dust in the Andromeda galaxy, M31, with unprecedented spatial resolution $(\sim 30 \mathrm{pc})$, column density accuracy, and constraints on the three-dimensional distributions of dust temperature and dust opacity index (hence grain size and composition), based on the new PPMAP Bayesian analysis procedure. We confirm the overall radial variation of dust opacity index reported by other recent studies, including the central decrease within $\sim 3 \mathrm{kpc}$ of the nucleus. We also investigate the detailed distribution of dust in the nuclear region, a prominent feature of which is an $\sim 500 \mathrm{pc}$ bar-like structure seen previously in $\mathrm{H} \alpha$. The nature of this feature has been the subject of some debate. Our maps show it to be the site of the warmest dust, with a mean line-of-sight temperature $\sim 30 \mathrm{~K}$. A comparison with the stellar distribution, based on 2MASS data, provides strong evidence that it is a gravitationally induced bar. A comparison with radial velocity maps suggests the presence of an inflow towards the nucleus from opposite directions along this bar, fed presumably by the nuclear spiral with which it appears to connect. Such behaviour is common in large-scale bars in spiral galaxies, as is the phenomenon of nested bars whereby a subkiloparsec nuclear bar exists within a large-scale primary bar. We suggest that M31 represents an example of such nesting.
\end{abstract}

Key words: techniques: high angular resolution-dust, extinction-galaxies: bulgesgalaxies: individual: M31 - galaxies: kinematics and dynamics - galaxies: spiral.

\section{INTRODUCTION}

High-resolution mapping of dust in spiral galaxies can provide information regarding star formation rates, the interstellar radiation field, and the global dynamics of the galaxies themselves. Such studies have been greatly aided by data from the Herschel Space Observatory whose wavelength coverage $(70-500 \mu \mathrm{m})$ includes the peak of the spectral range of cool dust emission. In terms of spatial resolution, the most favourable candidate for study is the Andromeda galaxy, M31, being our nearest spiral neighbour, at an estimated distance of $780 \mathrm{kpc}$ (Stanek \& Garnavich 1998; Villardell, Ribas \& Jordi 2006). Knowledge of the distribution of cool dust provides constraints on a number of physical phenomena in this galaxy and hence the Herschel observations represent an important component in the suite of multiwavelength data which extends from radio wavelengths to $\mathrm{X}$-rays.

The spatial structure of M31, as viewed in the far-infrared, is dominated by a set of bright concentric rings whose approximate radii, in order of decreasing prominence, are 10,5 , and $15 \mathrm{kpc}$ (Habing et al. 1984; Gordon et al. 2006; Fritz et al. 2012; Smith et al. 2012; Kirk et al. 2015; Lewis et al. 2015). The disc of the galaxy is inclined at $77^{\circ}$ to the plane of the sky, with a tilt axis

^E-mail: Ken.Marsh@astro.cf.ac.uk at a position angle $38^{\circ}$ (Fritz et al. 2012). There is also an inner ring, of approximate radius $0.7 \mathrm{kpc}$, which is less inclined than the main structure (Melchior \& Combes 2011, 2013) and which may represent the aftermath of a collision with the M32 dwarf galaxy (Block et al. 2006). Studies of the inner isophotes at optical and near-infrared wavelengths have provided evidence of a large-scale bar of length $\sim 10 \mathrm{kpc}$ [Athanassoula \& Beaton 2006; Beaton et al. 2007; Opitsch 2016; Diaz, Wegg \& Gerhard 2017, although the existence of such a bar has been questioned by Melchior \& Combes (2011)].

Evidence for significant radial variations in dust properties has been obtained by Smith et al. (2012) using fits of model spectral energy distributions (SEDs) to Herschel continuum data at six bands in the wavelength range 70-500 $\mu \mathrm{m}$. The angular spatial resolution was 36 arcsec, corresponding to a linear resolution of $\sim 140 \mathrm{pc}$ at the distance of M31. They found that the dust opacity index, $\beta$, increases inwards, reaching a maximum value at a radial distance $\sim 3 \mathrm{kpc}$, inside of which it then decreases towards the nucleus. A similar conclusion has been reached by Draine et al. (2014).

In this paper, we present the results of our investigation of the dust distribution in Andromeda using the same Herschel data as used by Smith et al. (2012), but processed using a new Bayesian analysis procedure, PPMAP (Marsh, Whitworth \& Lomax 2015), which yields significantly higher spatial resolution $(30 \mathrm{pc})$. This procedure, 
outlined in the next section, overcomes a number of limitations in standard analysis techniques. These include the requirement that all input images be degraded to the lowest resolution (normally 36 arcsec in the case of Herschel data) and the assumption that the dust has uniform temperature and composition along each line of sight. Such techniques return typically a pair of two-dimensional maps, one giving a representative dust temperature, $\hat{T}_{\mathrm{D}}(x, y)$, and the other an approximate line-of-sight column density, $\hat{N}(x, y)$. Here, $N$ is the total column density of material (dust plus gas), expressed in units of hydrogen molecule masses per unit area at the source, and the hat symbols (e.g. $\hat{N}$ ) denote values obtained with the standard analysis procedure.

Expressing the dust distribution in this way, i.e. in terms of column density, involves some grain model assumptions. Specifically, we need to assume a value for the opacity per unit mass at some reference wavelength which we take as $300 \mu \mathrm{m}$, as discussed in the next section. However, PPMAP can alternatively produce maps of optical depth at that wavelength, free from any grain model assumptions. The results could then be compared with optical and/or nearinfrared extinction measurements to extend the extinction curve to $300 \mu \mathrm{m}$ in a model-independent way. Such an analysis forms the basis of a companion paper (Whitworth \& Marsh, in preparation).

\section{METHODOLOGY}

In contrast with previous approaches to the dust mapping problem, the PPMAP procedure drops the assumptions of uniform dust temperature and opacity index along the line of sight (Marsh et al. 2015). ${ }^{1}$ After taking proper account of the point spread function and spectral response of the telescope, it returns a pair of four-dimensional hypercubes containing, respectively, the expectation values of differential column density,

$N_{T \beta} \equiv \frac{\partial^{2} N}{\partial T_{\mathrm{D}} \partial \beta_{\mathrm{D}}}$,

and their associated uncertainties, as a function of $\left(x, y, T_{\mathrm{D}}, \beta_{\mathrm{D}}\right)$. Here, $\beta_{\mathrm{D}}$ is the opacity index of the dust in the far-infrared, i.e.

$\beta_{\mathrm{D}}=-\frac{\mathrm{d} \log \kappa_{\lambda}}{\mathrm{d} \log \lambda}$

$\kappa_{\lambda}$ is the mass opacity coefficient of the dust at wavelength $\lambda$, and the derivative is evaluated in the wavelength interval covered by Herschel. Thus, $N_{T \beta}\left(x, y, T_{\mathrm{D}}, \beta_{\mathrm{D}}\right)$ is the best estimate of the lineof-sight column density at $(x, y)$, in unit dust temperature interval about $T_{\mathrm{D}}$ and unit opacity index interval about $\beta_{\mathrm{D}}$. The procedure assumes only that the emitting dust is in radiative equilibrium, and that, at the observed wavelengths, the emission is optically thin. It works by defining a grid of discrete values of $x, y, T_{\mathrm{D}}$, and $\beta_{\mathrm{D}}$, which represent sampling locations in the continuous state space of $\left(x, y, T_{\mathrm{D}}, \beta_{\mathrm{D}}\right)$. It then iterates towards the set of differential column densities, in the vicinities of those grid points, that best reproduces the observed monochromatic intensities. The resolution on the plane of the sky $(x, y)$ is increased by a factor of about 4.5 relative to the standard analysis procedure, yielding maps of angular resolution of $\sim 8$ arcsec, sampled by 4 arcsec square pixels.

The dust temperature sampling interval is arbitrary. For this work, we have used twelve representative dust temperatures that

\footnotetext{
${ }^{1}$ The original version treated the opacity index, $\beta_{\mathrm{D}}$, as constant. We have extended the algorithm by treating $\beta_{\mathrm{D}}$ as an additional state variable. Further details are given in Appendix A.
}

are equally spaced in logarithm between 10.0 and $50.0 \mathrm{~K}$, i.e. $T_{1}=$ $10.0 \mathrm{~K}, T_{2}=11.6 \mathrm{~K}, T_{3}=13.4 \mathrm{~K}, T_{4}=15.5 \mathrm{~K}, T_{5}=18.0 \mathrm{~K}, T_{6}=$ $20.8 \mathrm{~K}, T_{7}=24.1 \mathrm{~K}, T_{8}=27.8 \mathrm{~K}, T_{9}=32.2 \mathrm{~K}, T_{10}=37.3 \mathrm{~K}$, $T_{11}=43.2 \mathrm{~K}$, and $T_{12}=50.0 \mathrm{~K}$.

The opacity index sampling interval is also arbitrary, and we have used four representative values equally spaced linearly between $\beta_{\mathrm{D}}=1.5$ and $\beta_{\mathrm{D}}=3.0$, i.e. $\beta_{1}=1.5, \beta_{2}=2.0, \beta_{3}=2.5$, and $\beta_{4}=3.0$. If we were to invoke more representative dust temperatures and/or opacity indices, the computational cost of the analysis would increase proportionately but further accuracy would not necessarily be gained.

Our column density scale is based on an assumed opacity of $0.1 \mathrm{~cm}^{2} \mathrm{~g}^{-1}$ at a wavelength of $300 \mu \mathrm{m}$. The reference opacity is defined with respect to total mass (dust plus gas). Although observationally determined, it is consistent with a gas-to-dust ratio of 100 (Hildebrand 1983), and we use it here on the assumption that interstellar dust grains in M31 have similar properties to those in our own Galaxy.

Given the $N_{T \beta}$ hypercube, we can integrate out $\beta$ to obtain two three-dimensional data cubes containing, respectively, representative values of

$N_{T} \equiv \frac{\mathrm{d} N}{\mathrm{~d} T_{\mathrm{D}}}=\int_{\text {all } \beta_{\mathrm{D}}} \frac{\partial^{2} N}{\partial T_{\mathrm{D}} \partial \beta_{\mathrm{D}}} \mathrm{d} \beta_{\mathrm{D}}$

and their associated uncertainties. $N_{T}\left(x, y, T_{\mathrm{D}}\right)$ is the best estimate of column density along the line of sight at $(x, y)$, in unit dust temperature interval about $\sim T_{\mathrm{D}}$. This data cube can be interpreted in two interesting ways. First, we can take slices at the different representative dust temperatures, $T_{i}$, and plot separately the column density of dust in each dust temperature interval, $\left[\left(T_{i-1} T_{i}\right)^{1 / 2}\right.$ $\left.\leq T_{\mathrm{D}} \leq\left(T_{i} T_{i+1}\right)^{1 / 2}\right]$. Secondly, we can consider individual pixels separately, and analyse the mass-weighted distribution of dust temperature along the line of sight through the pixel. Since the dust temperature depends on the ambient radiant energy density, $U_{\mathrm{RAD}}$ (in relatively unshielded regions, $T_{\mathrm{D}} \propto U_{\mathrm{RAD}}^{0.20 \pm 0.05}$ ), this can help to resolve regions along the line of sight that have different ambient radiation fields.

Similarly, we can integrate out $T_{\mathrm{D}}$ to obtain two threedimensional data cubes containing, respectively, representative values of

$N_{\beta} \equiv \frac{\mathrm{d} N}{\mathrm{~d} \beta_{\mathrm{D}}}=\int_{\operatorname{all} T_{\mathrm{D}}} \frac{\partial^{2} N}{\partial T_{\mathrm{D}} \partial \beta_{\mathrm{D}}} \mathrm{d} T_{\mathrm{D}}$

and their associated uncertainties. $N_{\beta}\left(x, y, \beta_{\mathrm{D}}\right)$ is the best estimate of the line-of-sight column density at $(x, y)$, in unit opacity index interval about $\sim \beta_{\mathrm{D}}$. Again, this data cube can be interpreted in two interesting ways. First, we can take slices at the different representative opacity indices, $\beta_{j}$, and plot the column density of dust in each opacity index interval, $\left(\beta_{j}-0.25 \leq \beta_{\mathrm{D}} \leq \beta_{j}+0.25\right)$. Secondly, we can consider individual pixels separately, and analyse the massweighted distribution of opacity index along the line of sight through the pixel. Since the opacity index depends on the constitution of the dust (size, composition, shape), this can help to distinguish regions along the line of sight that have different chemical histories.

Next, we can integrate out both $\beta_{\mathrm{D}}$ and $T_{\mathrm{D}}$ to obtain two twodimensional maps of

$N=\int_{\text {all } T_{\mathrm{D}}} \frac{\mathrm{d} N}{\mathrm{~d} T_{\mathrm{D}}} \mathrm{d} T_{\mathrm{D}}$

and its associated uncertainty. Here, $N(x, y)$ gives the best estimate for the line-of-sight column density at $(x, y)$. 


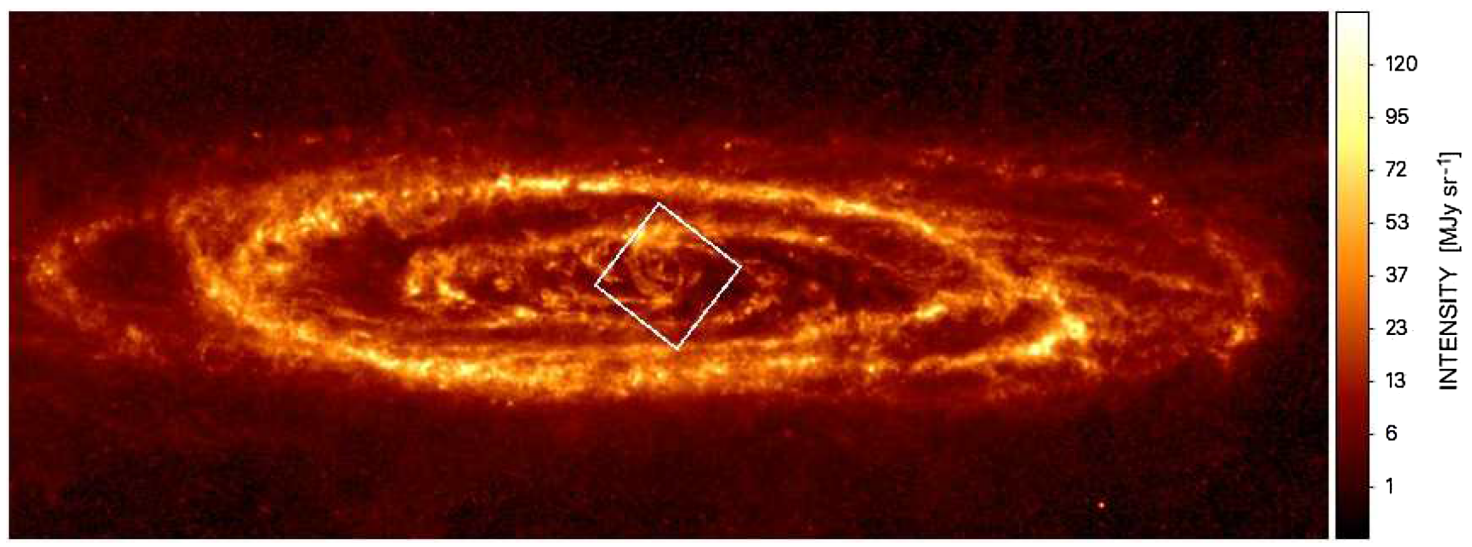

Figure 1. M31 as viewed in the $250 \mu \mathrm{m}$ band by the Herschel SPIRE instrument. The square box delineates the 'ZoomZone' discussed in the text. Its size is 700 arcsec on a side, corresponding to $2.65 \mathrm{kpc}$ based on an assumed distance of $780 \mathrm{kpc}$.

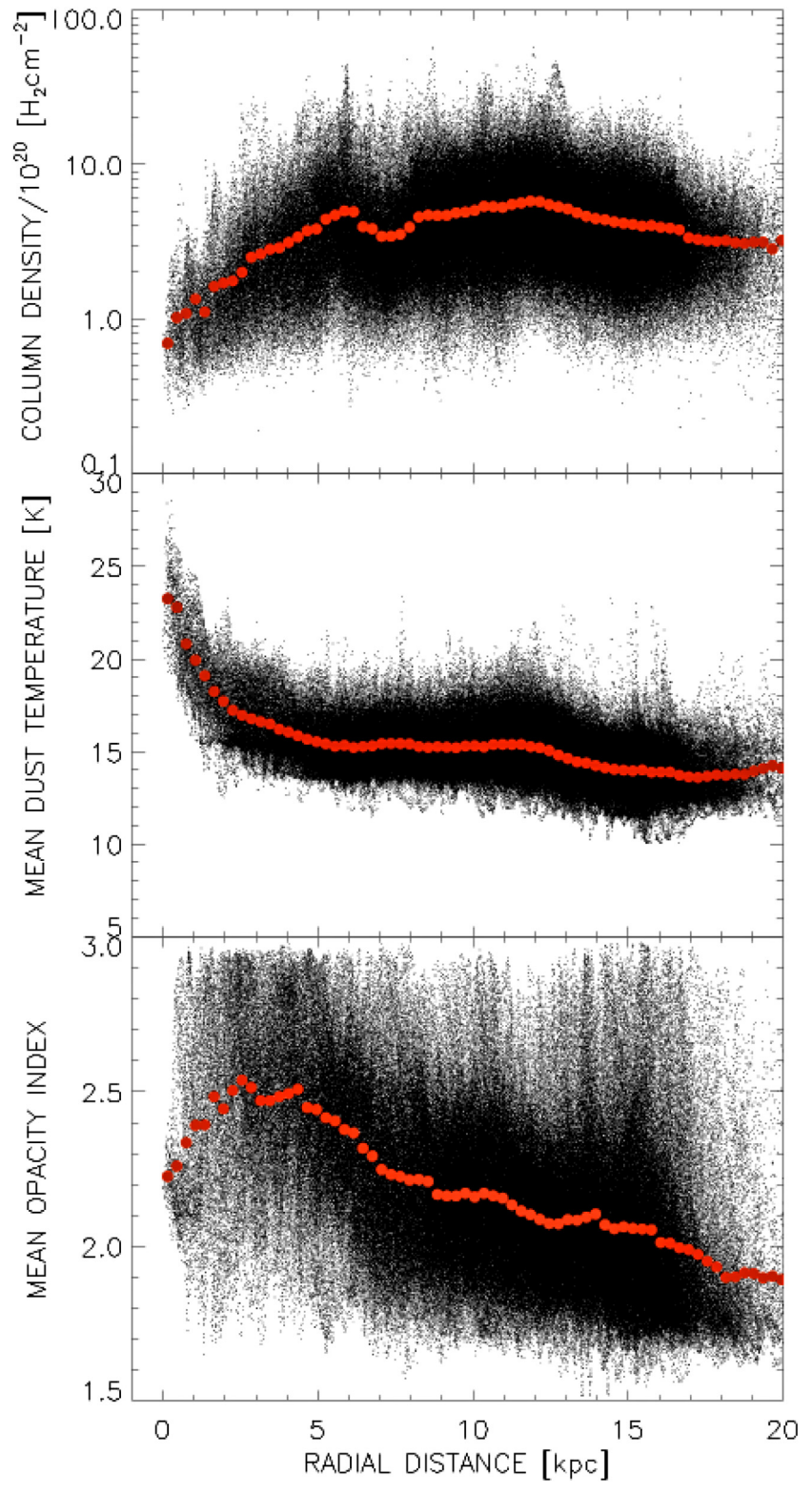

Figure 2. Radial variation of total line-of-sight column density, $N$, mean dust temperature, $\left\langle\bar{T}_{\mathrm{D}}\right\rangle$, and mean dust opacity index, $\left\langle\bar{\beta}_{\mathrm{D}}\right\rangle$. The red filled circles represent averages in $300 \mathrm{pc}$ bins.

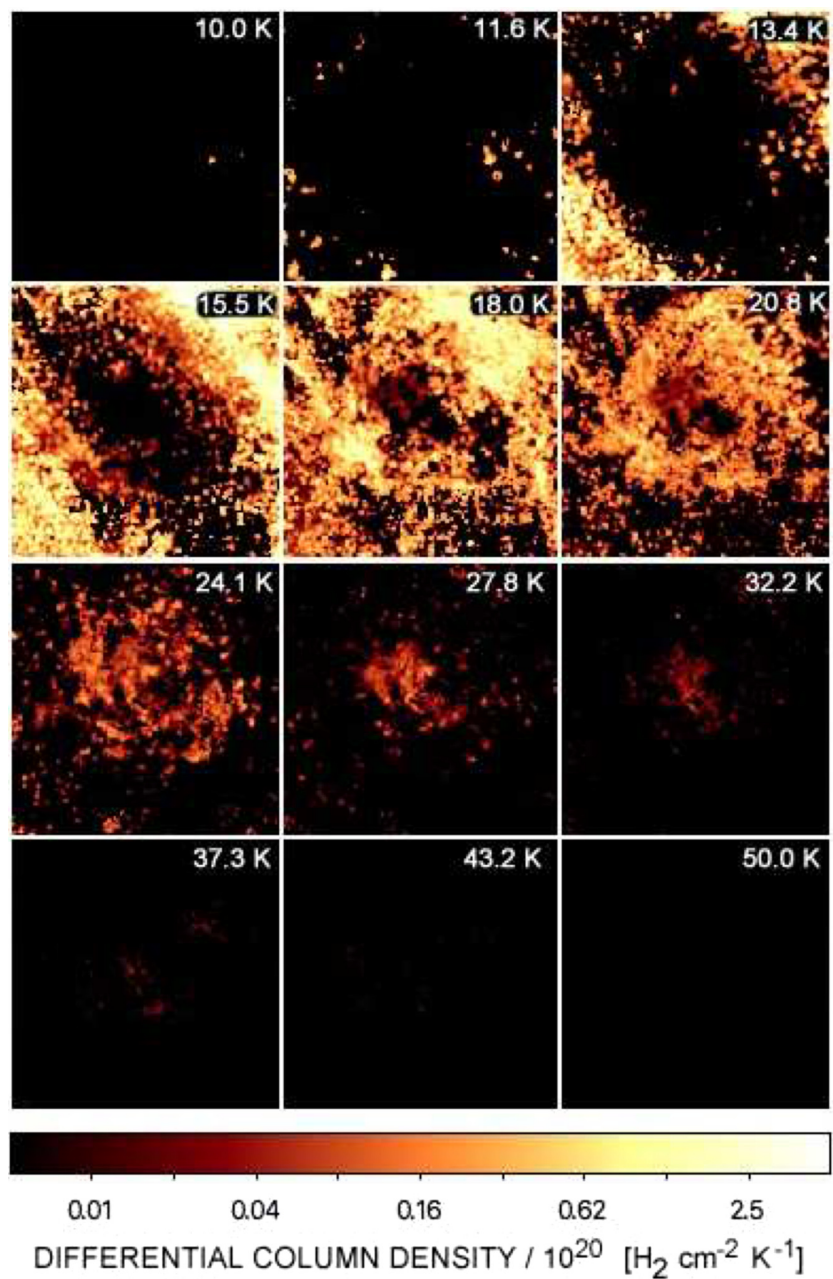

Figure 3. Maps of differential column density as a function of temperature, for the ZoomZone. These maps have been truncated at the $5 \sigma$ level. The field of view in each case is $700 \operatorname{arcsec}(2.65 \mathrm{kpc})$ square.

We can obtain an estimate of the mass-weighted mean dust temperature, $\bar{T}_{\mathrm{D}}(x, y)$, along the line of sight at $(x, y)$,

$\bar{T}_{\mathrm{D}}=\frac{1}{N} \int_{\text {all } T_{\mathrm{D}}} T_{\mathrm{D}} \frac{\mathrm{d} N}{\mathrm{~d} T_{\mathrm{D}}} \mathrm{d} T_{\mathrm{D}}$, 

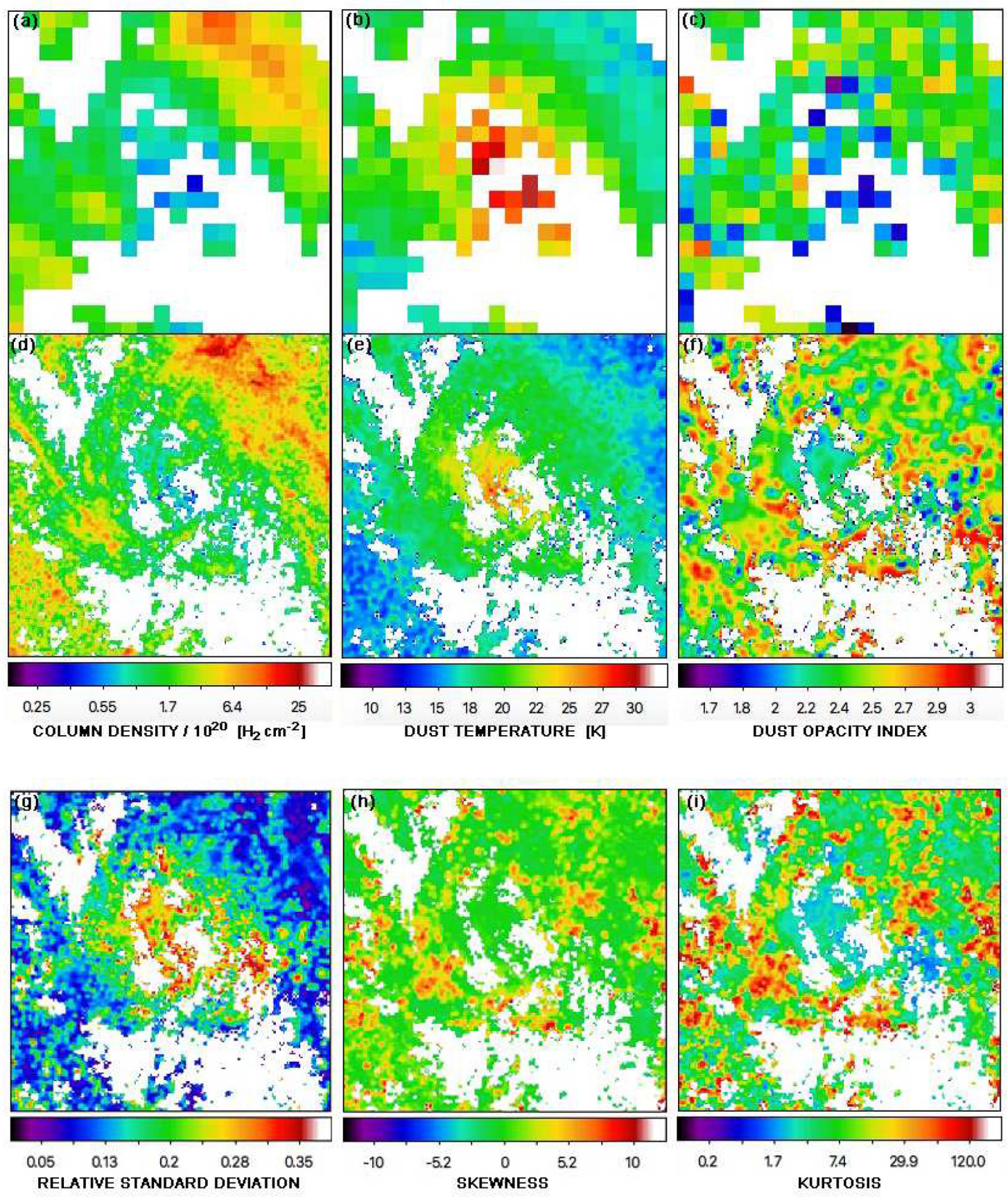

Figure 4. Maps of line-of-sight integrated quantities for the ZoomZone, each with a field of view of $700 \operatorname{arcsec}(2.65 \mathrm{kpc})$ square, and each truncated at the $5 \sigma$ level with respect to column density. Top row: Maps obtained by Smith et al. (2012) using the standard analysis procedure, viz. (a) integrated column density, $\hat{N}$, (b) mean dust temperature, $\hat{T}_{\mathrm{D}}$, and (c) mean dust opacity index, $\hat{\beta}_{\mathrm{D}}$. Middle row: The corresponding maps obtained with PPMAP, viz. (d) the integrated column density, $N$, (e) the mass-weighted mean dust temperature, $\bar{T}_{\mathrm{D}}$, and (f) the mass-weighted mean opacity index, $\bar{\beta}_{\mathrm{D}}$. Bottom row: Maps of the higher order moments of the dust temperature distribution, viz. (g) the standard deviation, $\sigma_{T}$, expressed as a fraction of the local mean temperature, $\bar{T}_{\mathrm{D}}$, (h) the skewness, and (i) the kurtosis.

and higher order moments of the dust temperature distribution along the line of sight (standard deviation, skewness, and kurtosis), as discussed below.

Finally, we can obtain an estimate of the mass-weighted mean opacity index, $\bar{\beta}_{\mathrm{D}}(x, y)$, along the line of sight at $(x, y)$ using an integral analogous to equation (5).

\section{OBSERVATIONS}

M31 was observed using the Herschel PACS and SPIRE instruments which provided continuum images in bands centred on wavelengths of 70, 100, 160, 250, 350, and $500 \mu \mathrm{m}$. Full details of the observing strategy, calibration, and map-making procedures are given by Fritz 

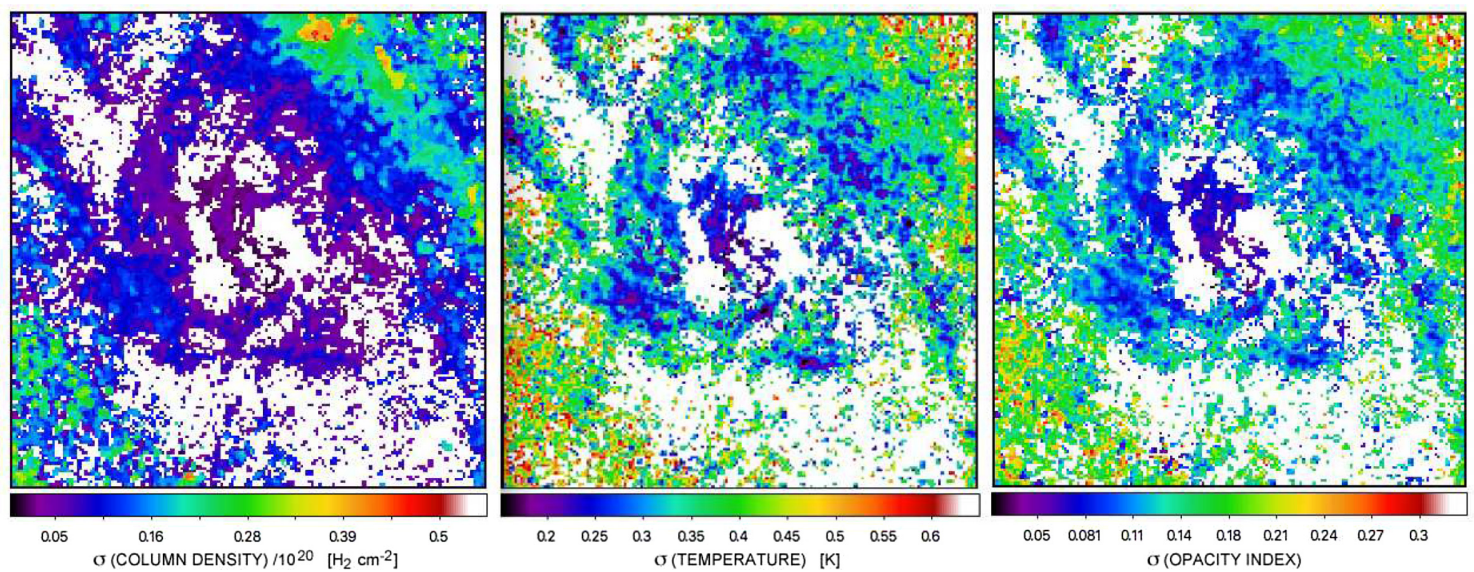

Figure 5. Uncertainty maps of column density, temperature, and opacity index for the ZoomZone, each with a field of view of 700 arcsec (2.65 kpc) square. They represent the uncertainty values corresponding to the maps in the middle row of Fig. 4.

et al. (2012) and Smith et al. (2012). As an example, Fig. 1 shows a SPIRE image of M31 in the $250 \mu \mathrm{m}$ band.

The spatial resolution values of the scan maps, i.e. the beam sizes at full width at half-maximum (FWHM), are approximately $8.5,12.5,13.3,18.2,24.5$, and 36.0 arcsec at the six wavelengths, respectively. For our analysis, we use PSFs based on the measured Herschel beam profiles (Poglitsch et al. 2010; Griffin et al. 2013).

\section{RESULTS}

The above data have been used to generate a 4D hypercube of differential column density as a function of sky location, dust temperature, and dust opacity index, from which we can derive various line-ofsight integrated quantities such as the integrated column density of dust plus gas, $N$, the mean dust temperature, $\bar{T}_{\mathrm{D}}$, and the mean dust opacity index, $\bar{\beta}_{\mathrm{D}}$. Here, and in the following discussion, an overscore (e.g. $\bar{T}_{\mathrm{D}}$ and $\bar{\beta}_{\mathrm{D}}$ ) denotes mass-weighted averages along the line of sight.

Fig. 2 shows the radial variation of azimuthally averaged values of these quantities, $N,\left\langle\bar{T}_{\mathrm{D}}\right\rangle$, and $\left\langle\bar{\beta}_{\mathrm{D}}\right\rangle$, at each galactocentric radius. Galactocentric radii are estimated assuming that the mid-plane of Andromeda makes an angle of $\theta=77^{\circ}$ with the plane of the sky, and that the position angle of the tilt axis is $38^{\circ}$. We note that the radial plot of $\left\langle\bar{\beta}_{\mathrm{D}}\right\rangle$ in this figure indicates a central dip in the dust opacity index, consistent with previous reports by Smith et al. (2012) and Draine et al. (2014). In particular, there is detailed correspondence with fig. 13 of Draine et al. (2014), including the local maximum at $r \sim 3 \mathrm{kpc}$.

\subsection{The central region: 'ZoomZone'}

In the remainder of the paper, we focus on the small region of Andromeda that is marked with a square of width $700 \operatorname{arcsec}(2.65 \mathrm{kpc})$ on Fig. 1, and refer to it as the ZoomZone. It is chosen because it includes the circumnuclear environment, the site of some interesting physical processes that are not well understood (see for example, Li, Wang \& Wakker 2009). Fig. 3 shows maps of temperaturedifferential column density, $N_{T}$, in this region, at all 12 temperatures. These maps, and the associated uncertainty maps, are available in the online material as described in Appendix B.

On the first (top) row of Fig. 4 are maps of (a) $\hat{N}$, (b) $\hat{T}_{\mathrm{D}}$, and (c) $\hat{\beta}_{\mathrm{D}}$ obtained by Smith et al. (2012) using the standard analysis procedure, cropped at the boundaries of the ZoomZone. The second (middle) row shows the corresponding maps obtained with PPMAP, viz. (d) the integrated column density, $N$, (e) the mass-weighted mean dust temperature, $\bar{T}_{\mathrm{D}}$, and (f) the mass-weighted mean opacity index, $\bar{\beta}_{\mathrm{D}}$. These maps illustrate the greatly increased resolution obtained with PPMAP. In addition, they demonstrate the ability of PPMAP to dig out large quantities of cold dust that is underestimated by the standard analysis procedure. This is illustrated by the presence of lower mean dust temperatures (by up to $2 \mathrm{~K}$ ) in panel (e) than panel (b), while Fig. 3 shows the presence of significantly cooler dust $(\sim 10 \mathrm{~K}$ as opposed to $\sim 15 \mathrm{~K})$ than indicated by the standard analysis in the corresponding region. As a consequence, in many areas PPMAP obtains a lower mean dust temperature $\left(\bar{T}_{\mathrm{D}}<\hat{T}_{\mathrm{D}}\right)$ and a higher net column density $(N>\hat{N})$. The third (bottom) row shows $(\mathrm{g})$ the relative standard deviation, $\sigma_{T} / \bar{T}_{\mathrm{D}}$, (h) the skewness, $\operatorname{Skew}(T)$, and (i) the kurtosis, $\operatorname{Kurt}(T)$ of the dust temperature distribution along each line of sight. These three quantities are defined by

$\sigma_{T}=\left(\frac{1}{N} \int_{\text {all } T_{\mathrm{D}}} \frac{\mathrm{d} N}{\mathrm{~d} T_{\mathrm{D}}}\left(T_{\mathrm{D}}-\bar{T}_{\mathrm{D}}\right)^{2} \mathrm{~d} T_{\mathrm{D}}\right)^{1 / 2}$

$\operatorname{Skew}(T)=\left(\frac{1}{N} \int_{\text {all } T_{\mathrm{D}}} \frac{\mathrm{d} N}{\mathrm{~d} T_{\mathrm{D}}}\left(T_{\mathrm{D}}-\bar{T}_{\mathrm{D}}\right)^{3} \mathrm{~d} T_{\mathrm{D}}\right) / \sigma_{T}^{3}$

$\operatorname{Kurt}(T)=\left(\frac{1}{N} \int_{\text {all } T_{\mathrm{D}}} \frac{\mathrm{d} N}{\mathrm{~d} T_{\mathrm{D}}}\left(T_{\mathrm{D}}-\bar{T}_{\mathrm{D}}\right)^{4} \mathrm{~d} T_{\mathrm{D}}\right) / \sigma_{T}^{4}$.

They provide information on the form of the temperature distribution along each line of sight. In particular, for a pure Gaussian distribution, we would expect a skewness of zero and a kurtosis of 3. Examination of Fig. 4 shows that these conditions are met over much of the ZoomZone, although there are localized regions in which the kurtosis is large ( $\gtrsim 100$ in many cases). Large values of the kurtosis indicate the presence of a significant number of high-temperature outliers in the tail of the distribution. It is not clear what high kurtosis implies physically; a comparison with published positions of planetary nebulae (Merrett et al. 2006), X-ray sources (Stiele et al. 2011), and supernova remnants (Lee \& Lee 2014) indicates little or no correspondence with high-kurtosis features in the ZoomZone, while none of the $\mathrm{H}$ II regions listed by Azimlu, Marciniak \& Barmby (2011) falls within that zone. However, a comparison over the full field of M31 shows a high degree 

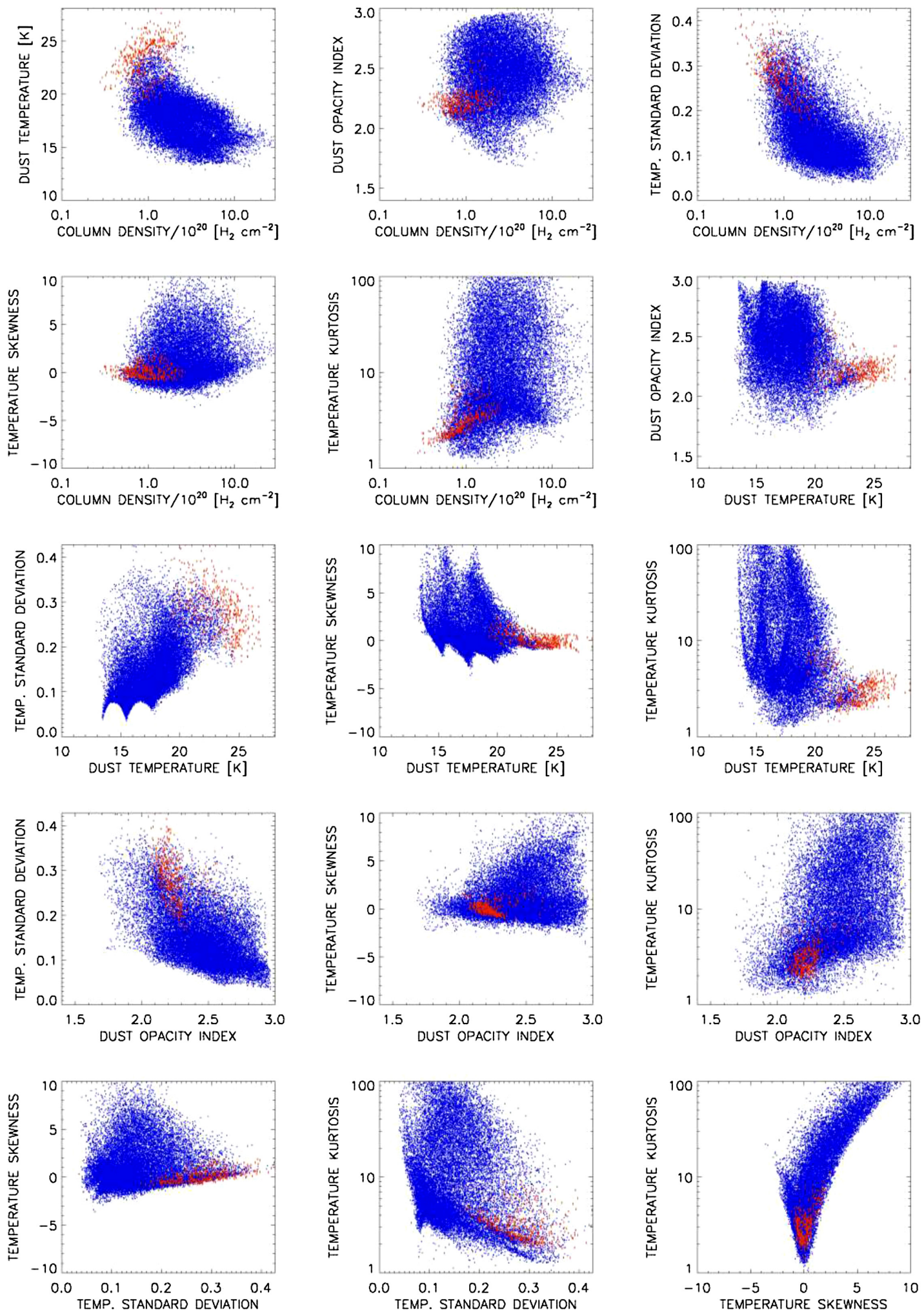

Figure 6. Scatter plots of line-of-sight integrated quantities for the ZoomZone. The standard deviation of temperature is expressed as a fraction of the local mean line-of-sight temperature. The red points represent spatial locations within the central bar-like feature in the nuclear spiral, within $270 \mathrm{pc}$ of the nucleus. The spiky lower envelopes in the plots of $\sigma_{T}$ and $\operatorname{Skew}(T)$ versus $T_{\mathrm{D}}$ are due to the discretization of temperature values in PPMAP.

of correlation with the locations of $\mathrm{H}$ II regions from the Azimlu et al. (2011) survey, in the sense that $\mathrm{H}$ II regions are preferentially located in regions of high temperature-kurtosis and, interestingly, low standard deviation of temperature. This suggests that high kur- tosis might be a signpost of star formation, and this is confirmed by a comparison with a map of star formation rate (Ford et al. 2013). It would clearly be interesting to investigate what kurtosis and the other temperature moments tell us about star formation, but since 


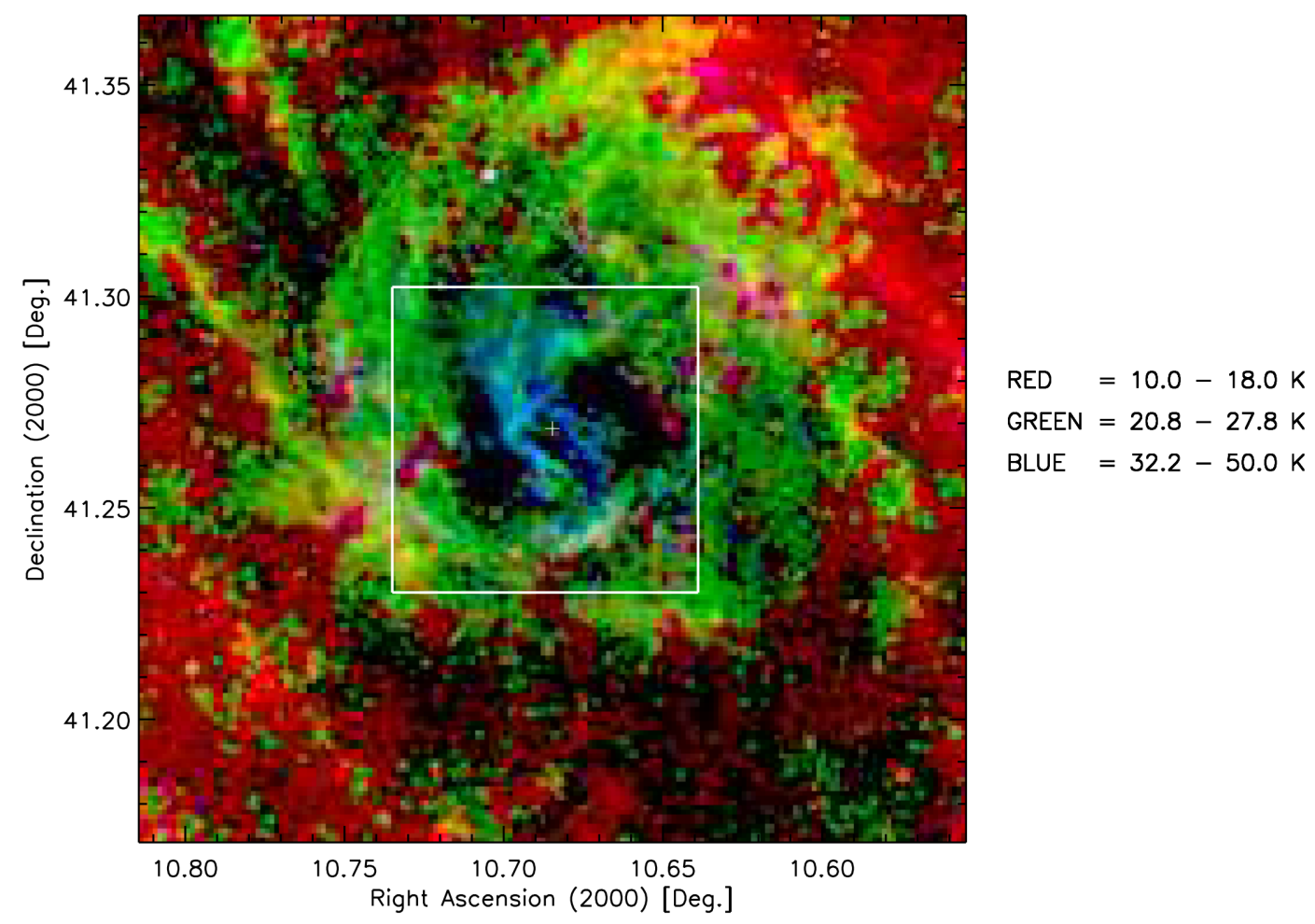

Figure 7. Three-temperature composite of the ZoomZone, where the dust temperature ranges correspond to the colours indicated. The white square delineates the inner portion corresponding to the field of view of the extinction map of Dong et al. (2016).

the current focus is on the nuclear region, we defer a discussion of more global phenomena to a future paper.

The uncertainty maps corresponding to the middle row of Fig. 4 (column density, temperature, and opacity index) are presented in Fig. 5.

Fig. 6 shows a set of scatter plots, for the ZoomZone, between all pairs of the six quantities, i.e. column density, temperature, opacity index, and temperature standard deviation, skewness, and kurtosis. Various correlations are apparent, including an anticorrelation between kurtosis and standard deviation which, as mentioned above, appears to be a global characteristic. Also, particularly relevant here are the correlations involving the nuclear bar-like feature, plotted in red. This feature clearly shows some systematic behaviour, one example being that the dust opacity index, $\bar{\beta}_{\mathrm{D}}(x, y)$, is positively correlated with dust temperature in the interior of the nuclear spiral (within $270 \mathrm{pc}$ of the nucleus). This goes against the larger scale trend in which $\bar{\beta}_{\mathrm{D}}(x, y)$ is anticorrelated with $\bar{T}_{\mathrm{D}}$ within the central $\sim 3 \mathrm{kpc}$. As discussed by Smith et al. (2012), various explanations are possible for that anticorrelation, such as the destruction of grain ice-mantles in the increasingly intense interstellar radiation field towards the centre of the galaxy. However, the fact that it switches to a positive correlation near the very centre would seem to argue against that explanation, although it might instead indicate that a separate population of grains is present.

Fig. 7 represents a three-temperature composite for the ZoomZone. It shows that the innermost portion is dominated by a bar-like feature which appears to consist of a pair of hot, roughly parallel, strands with a central crossover. It is distinctly visible at other wavelengths, such as $\mathrm{H} \alpha$ (Jacoby, Ford \& Ciardullo 1985) and $70 \mu \mathrm{m}$ (in our Herschel image), although less so at $24 \mu \mathrm{m}$ (Gordon et al. 2006) due to confusion by a dense concentration of bulge stars. Its nature has been the subject of debate, but with a length of only $\sim 500 \mathrm{pc}$, it clearly does not correspond to the much larger $(\sim 10 \mathrm{kpc})$ bar inferred from near-infrared morphology and dynamical simulations (Athanassoula \& Beaton 2006).

\subsection{The nature of the bar-like feature}

Some information on the 3D geometry of the bar-like feature can be obtained from a comparison of the column density distribution derived here on the basis of dust emission, and a previously published extinction map (Dong et al. 2016), as shown in panels (a) and (b) of Fig. 8. These maps show some distinct differences. For example, the filamentary structure that extends between reference points ' $A$ ' and ' $\mathrm{B}$ ' in the column density map is absent in the extinction map, suggesting that it lies behind the bulge and hence behind the source of illumination which enables foreground features to be detected in extinction. This behaviour was also noted by Dong et al. (2016), who identify the feature with a dusty clump detected in $\mathrm{CO}$ and conclude, similarly, that it is located on the far side of the bulge. We will thus refer to the feature in question as the 'rear filament'. However, an adjacent filament that extends from the nucleus (' + ') to point ' $C$ ' is seen prominently in both emission and absorption, indicating that it is closer to us. This segment is part of a longer filament which passes directly over the nucleus in line-of-sight projection. We refer to this filament as the 'central filament'. Based on its elevated dust temperature (see Fig. 4e), it likely passes directly through the centre of the bulge where the interstellar radiation field due to evolved giant stars is strongest (Groves et al. 2012). Its overall position angle is $\sim 50^{\circ}$, although it contains a central 'jog' whereby it runs approximately east-west over the nucleus.

If the central filament is the manifestation of a bar, representing orbital motion under the influence of a bar-like gravitational potential (see for example, Sellwood \& Wilkinson 1993), we would 

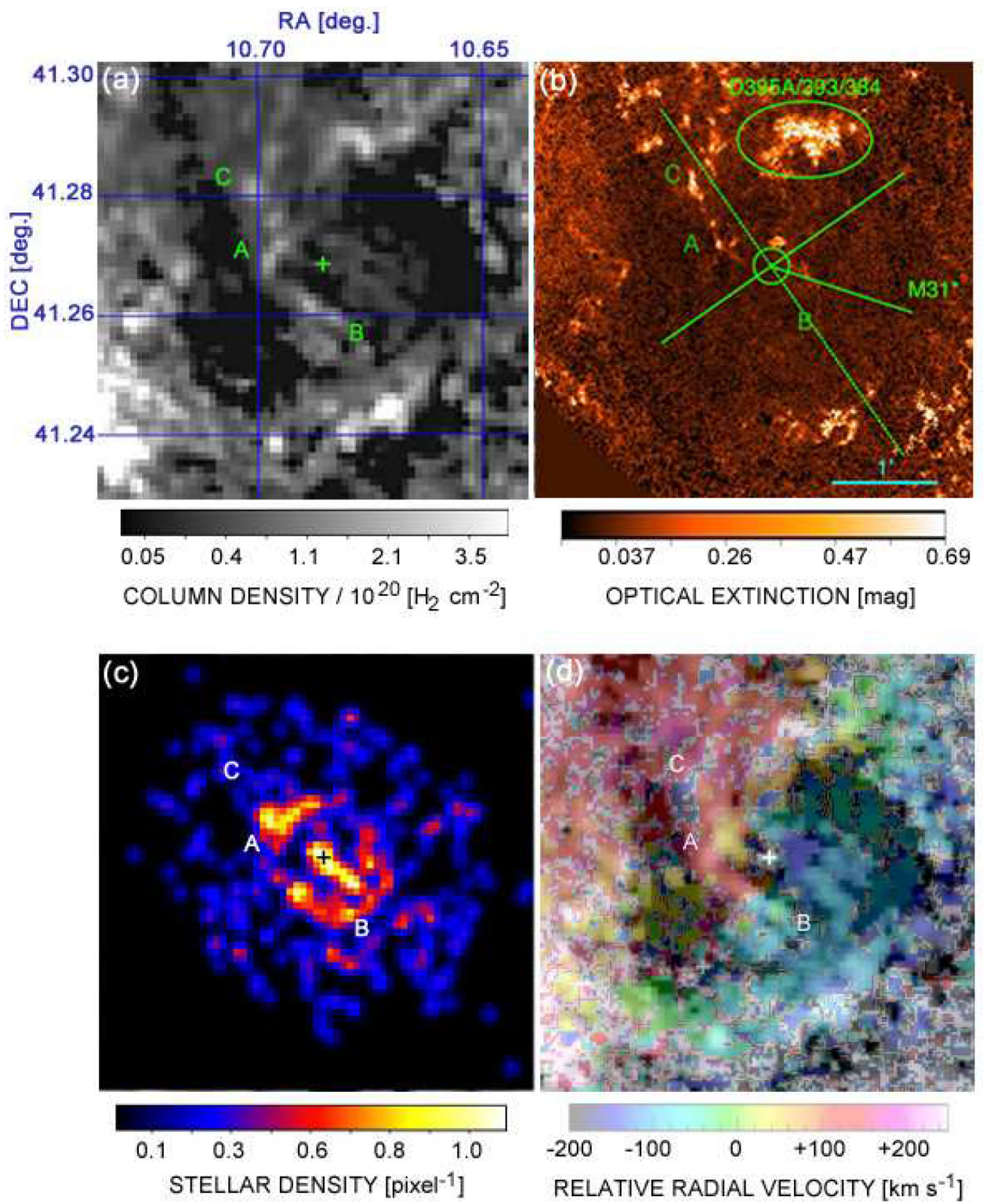

Figure 8. Comparison of column density, extinction, stellar density, and radial velocity distributions for the central bulge region of M31, as delineated by the white square in Fig. 7. (a) Total column density; (b) Optical extinction at a wavelength of $5447 \AA$, from Dong et al. (2016); (c) Stellar density in units of stars per pixel, representing the number density of 2MASS sources with $J \leq 14$, smoothed by a Gaussian kernel of FWHM=2 pixels ( 8 arcsec); (d) Radial velocity with respect to the systemic value of $300 \mathrm{~km} \mathrm{~s}^{-1}$, superposed on the total column density distribution. The radial velocities are from [N II] observations published previously by Boulesteix et al. (1987). In panels (a), (c), and (d), the position of the nucleus is marked by a plus sign (+). In (b), it is labelled as M31*. 'A', 'B', and 'C' are reference points discussed in the text.

expect to see some evidence of it in the distribution of bulge stars. Panel (c) of Fig. 8 shows that this is indeed the case - the bar structure is clearly visible in a map of stellar density. The latter represents the number density of 2 MASS $^{2}$ sources brighter than 14th magnitude at $J$ band, estimated using a circular Gaussian kernel whose width (FWHM) corresponds to 2 pixels $(8 \operatorname{arcsec})$. The position angle of the stellar bar is approximately $45^{\circ}$, reasonably consistent with that of the central filament. We thus interpret the central dust structure as a bar and explain its apparently double appearance in the column density map as a line-of-sight juxtaposition with the 'rear filament' which is actually located further back.

\footnotetext{
${ }^{2}$ Two Micron All Sky Survey (Skrutskie et al. 2006)
}

Further elucidation of the nature of the central structure can be obtained from a comparison with radial velocity information. Since the morphology of the dust distribution closely resembles that of the ionized gas ( $\mathrm{Li}$ et al. 2009), we make use of radial velocities of [N $\mathrm{II}$ ], published previously by Boulesteix et al. (1987). Such a comparison is facilitated by panel (d) of Fig. 8, which represents a superposition of the [N II] radial velocities on the dust column density distribution. The general trend of the radial velocities (red-shifted at the upper left and blue-shifted at the lower right) is consistent with the general rotational motion in the kinematic model presented by Melchior \& Combes (2011), involving an inner disc tilted at a position angle of $70^{\circ}$ and an inclination of $43^{\circ}$ to the plane of the sky. For the reasons discussed above, we now believe that this disc is actually a compact spiral containing a classical bar. 
Fig. 8(d) also shows that the profile of radial velocity along the central filament is quite distinct from that of the general rotational motion. It changes abruptly from a red-shift to a blue-shift as the nucleus position is traversed, at which point the velocity gradient is approximately east-west, consistent with the local orientation of the jog in the filament. This velocity structure, together with the apparent positional coincidence between the central filament and nucleus, would indicate motion either towards or away from the nucleus. However, we can eliminate the latter possibility because of the orientation - the lower right portion of the filament must be pointed away from us since it experiences more optical extinction than the upper left portion. Such an orientation is, in fact, consistent with the inferred tilt of the nuclear spiral as a whole, based on the Melchior \& Combes (2011) kinematic model. We thus conclude that the red- and blue-shifts indicate motion towards the nucleus, i.e. that the filament constitutes an accretion channel. Since the filament appears to connect with the nuclear spiral at diametrically opposite points, it seems likely that these spiral arms provide the source of the inflow.

We can estimate the inflow rate given the column density of the central filament $\left(\sim 2 \times 10^{20} \mathrm{H}_{2}\right.$ masses per $\left.\mathrm{cm}^{2}\right)$, the radial velocity $\left(\sim 100 \mathrm{~km} \mathrm{~s}^{-1}\right)$, the inclination $\left(\sim 47^{\circ}\right.$ to the line of sight $)$, and an estimated width of $8 \operatorname{arcsec}(60 \mathrm{pc})$. We thereby obtain a total flow rate (from both sides) of $\sim 0.05 \mathrm{M}_{\odot} \mathrm{yr}^{-1}$. This value greatly exceeds, by 8 orders of magnitude, the minimum accretion rate (based on mass-energy equivalence) required to power the central X-ray source, the estimated luminosity of which is $\simeq 1.2 \times 10^{36} \mathrm{erg} \mathrm{s}^{-1}$ (Li et al. 2009). It also exceeds, by 3 orders of magnitude, the Bondi accretion rate estimated by Li et al. It is, however, close to the inflow rate required for continuous replenishment of the hot corona according to the evaporation model of Li et al.

The role of the bar as a conduit for gas flow inwards from the spiral arms to the nucleus in barred galaxies has been established observationally (Knapen, Pérez-Ramírez \& Laine 2002) and has been the subject of a number of theoretical and modelling studies in which the inflow is attributed to bar-induced torques (see for example, García-Burillo et al. 2009; Hopkins \& Quataert 2011; Querejeta et al. 2016). It is not uncommon for nesting of bars to occur, whereby a subkiloparsec (secondary) nuclear bar exists within a large-scale primary bar (Laine et al. 2002; Shlosman \& Heller 2002; Wozniak 2015). We suggest that M31 represents an example of such nesting. Future dynamical modelling will be needed to determine whether our inferred nuclear bar can supply sufficient torque to drive the suggested inflow, and to establish the dynamical relationship between the primary and secondary bars.

\section{ACKNOWLEDGEMENTS}

We thank Anne-Laure Melchior for interesting discussions on this work. We gratefully acknowledge the support of a consolidated grant (ST/K00926/1), from the UK Science and Technology Funding Council. This work was performed using the computational facilities of the Advanced Research Computing at Cardiff (ARCCA) Division, Cardiff University. This publication makes use of data products from the Two Micron All Sky Survey, which is a joint project of the University of Massachusetts and the Infrared Processing and Analysis Center/California Institute of Technology, funded by the National Aeronautics and Space Administration and the National Science Foundation.

\section{REFERENCES}

Athanassoula E., Beaton R. L., 2006, MNRAS, 370, 1499

Azimlu M., Marciniak R., Barmby P., 2011, ApJ, 142, 139

Beaton R. L. et al., 2007, ApJ, 658, L91

Block D. L. et al., 2006, Nature, 443, 832

Boulesteix J., Georgelin Y. P., Lecoarer E., Marcelin M., Monnet G., 1987, A\&A, 178, 91

Diaz M. B., Wegg C., Gerhard O., 2017, MNRAS, 466, 4279

Dong H. et al., 2016, MNRAS, 459, 2262

Draine B. T. et al., 2014, ApJ, 780, 172

Ford G. P. et al., 2013, ApJ, 769, 55

Fritz J. et al., 2012, A\&A, 546, 34

Galliano F., 2018, MNRAS, 476, 1445

García-Burillo S. et al., 2009, A\&A, 496, 85

Gordon K. D. et al., 2006, ApJ, 638, L87

Griffin M. J. et al., 2013, MNRAS, 434, 992

Groves B. et al., 2012, MNRAS, 426, 892

Habing H. J. et al., 1984, ApJ, 278, L59

Hildebrand R. H., 1983, QJRAS, 24, 267

Hopkins P. F., Quataert E., 2011, MNRAS, 415, 1027

Jacoby G. H., Ford H., Ciardullo R., 1985, ApJ, 290, 136

Juvela M., Montillaud J., Ysard N., Lunttila T., 2013, A\&A, 556, A63

Kelly B. C., Shetty R., Stutz A. M., Kauffmann J., Goodman A. A., Launhardt R., 2012, ApJ, 752, 55

Kirk J. M. et al., 2015, ApJ, 798, 58

Knapen J. H., Pérez-Ramírez D., Laine S., 2002, MNRAS, 337, 808

Laine S., Shlosman I., Knapen J. H., Peletier R. F., 2002, ApJ, 567, 97

Lee J. H., Lee M. G., 2014, ApJ, 786, 130

Lewis A. R. et al., 2015, ApJ, 805, 183

Li Z., Wang Q. D., Wakker B. P., 2009, 397, 148

Marsh K. A., Whitworth A. P., Lomax O., 2015, MNRAS, 454, 4282

Melchior A.-L., Combes F., 2011, A\&A, 536, A52

Melchior A.-L., Combes F., 2013, A\&A, 549, A27

Merrett H. R. et al., 2006, MNRAS, 369, 120

Opitsch M., 2016, The Bar of the Andromeda Galaxy Revealed by Integral Field Spectroscopy. Ludwig-Maximilians-Universität München, München, Germany

Poglitsch A. et al., 2010, A\&A, 518, L2

Querejeta M. et al., 2016, A\&A, 588, 33

Sellwood J. A., Wilkinson A., 1993, Rep. Prog. Phys., 56, 173

Shlosman I., Heller C. H., 2002, ApJ, 565, 921

Skrutskie M. F., et al., 2006, AJ, 131, 1163

Smith M. W. L. et al., 2012, ApJ, 756, 40

Stanek K. Z., Garnavich P. M., 1998, ApJ, 503, L131

Stiele H., Pietsch W., Haberl F., Hatzidimitriou D., Barnard R., Williams B.

F., Kong A. K. H., Kolb U., 2011, A\&A, 534, A55

Villardell F., Ribas I., Jordi C., 2006, A\&A, 459, 321

Wozniak H., 2015, A\&A, 575, A7

\section{APPENDIX A: EXTENSION OF PPMAP TO ENABLE $\beta$ ESTIMATION}

The original version of PPMAP (Marsh et al. 2015) treated the opacity index, $\beta_{\mathrm{D}}$, as constant. We have extended the algorithm by treating $\beta_{\mathrm{D}}$ as an additional state variable, thus enabling the generation of $4 \mathrm{D}$ hypercubes of differential column density as a function of RA, Dec, $T_{\mathrm{D}}$, and $\beta_{\mathrm{D}}$. In doing so, it is necessary to guard against the well-known degeneracy between $T_{\mathrm{D}}$ and $\beta_{\mathrm{D}}$, whereby the presence of measurement noise induces an anticorrelation between the estimates of those quantities. Various approaches have been proposed for dealing with this problem, most of which are oriented towards fitting the observed SEDs of models of multiple uniform clumps in a given field. In the hierarchical Bayesian approach (Kelly et al. 2012; Galliano 2018), the solution is constrained by the use of a prior distribution designed to represent the statistics of the source pop- 
ulation as a whole and whose parameters are estimated adaptively from the observations. The algorithm then works towards finding solutions which minimize the dispersion of the estimated parameter values, and appears to work well provided one can characterize the source population by a single mode. However, extending the hierarchical Bayesian scheme to the more general imaging problem would present difficulties since it would belong in the category of single-pixel SED fitting techniques requiring all input images to be smoothed to the same resolution, thus losing an important advantage of PPMAP. In addition, one would need to assume a single temperature and opacity index along each line of sight and this, in itself, leads to difficulties in deducing dust properties (Juvela et al. 2013). The PPMAP procedure is not subject to that restriction.

We can, however, achieve the main goal of suppressing the $T_{\mathrm{D}^{-}}$ $\beta_{\mathrm{D}}$ degeneracy using a non-hierarchical Bayesian scheme in which we specify a priori the population statistics regarding $\beta_{\mathrm{D}}$. For this purpose, we assume that the a priori probability distribution of possible $\beta_{\mathrm{D}}$ values is described by a Gaussian function characterized by mean and standard deviation $\bar{\beta}$ and $\sigma_{\beta}$, respectively. The estimation procedure is then essentially the same as described in Marsh et al. (2015), except as noted below. As before, the desired differential column density, represented by vector $\rho$, is obtained numerically by a stepwise integration of the differential equation:

$\frac{\partial \rho}{\partial t}+\phi_{1} \rho=0$

where $\phi_{1}$ is the conditioning factor that contains information on both the measurement model and the data, and $t$ is a progress variable that increases from 0 to 1 during the solution procedure. The only modification necessary for incorporating the prior information regarding $\beta_{\mathrm{D}}$ is that the initial condition, $\rho_{(t=0)}=\eta$, is now replaced with

$\rho_{n}(t=0)=\eta \times \alpha \exp \left[-\left(\beta_{n}-\bar{\beta}\right)^{2} /\left(2 \sigma_{\beta}^{2}\right)\right]$

in which the index $n$ refers to the $n$th cell in the state space for which the local value of $\beta_{\mathrm{D}}$ is equal to $\beta_{n}$, and $\alpha$ is a normalization constant designed to ensure that the average value of $\rho_{n}(t=0)$ over all $n$ is equal to $\eta$. The latter quantity represents the a priori dilution, as defined by Marsh et al. (2015), for which we have assumed a value of 0.3 in our M31 analysis.
For the Gaussian prior on $\beta_{\mathrm{D}}$, we have assumed $\bar{\beta}=2.0$ and $\sigma_{\beta}=0.35$. In regions where the signal-to-noise ratio is high, these parameters have essentially no effect on the solution, so that no bias results (see for example, Galliano 2018), and this is true of both the hierarchical and non-hierarchical Bayesian approaches. However, in regions of low signal to noise, the algorithm will default to the prior itself so that, in essence, we are taking $\beta_{\mathrm{D}}$ to be 2.0 except where the data compel us otherwise.

\section{APPENDIX B: SUPPLEMENTARY MATERIAL}

We have made available online ${ }^{3}$ the set of PPMAP output files for M31. They consist of a set of files in FITS format which include:

(i) 4D hypercube of differential column density, with axes RA, Dec, dust temperature $\left(T_{\mathrm{D}}\right)$, and dust opacity index $\left(\beta_{\mathrm{D}}\right)$. The sets of grid values of $T_{\mathrm{D}}$ and $\beta_{\mathrm{D}}$ are specified in the FITS header.

(ii) $4 \mathrm{D}$ hypercube of $1 \sigma$ uncertainty values of differential column density4D hypercube of $1 \sigma$ uncertainty values of differential column density.

(iii) $2 \mathrm{D}$ map of integrated line-of-sight column density in units of $10^{20} \mathrm{H}_{2}$ molecules $\mathrm{cm}^{-2}$, truncated at the $5 \sigma$ level.

(iv) 2D map of mean line-of-sight dust opacity index, truncated at the $5 \sigma$ level.

(v) 2D map of mean line-of-sight dust temperature, truncated at the $5 \sigma$ column density level.

(vi) 2D map of the standard deviation of dust temperature along the line of sight, truncated at the $5 \sigma$ column density level.

(vii) $2 \mathrm{D}$ map of the skewness of the dust temperature distribution along the line of sight, truncated at the $5 \sigma$ column density level.

(viii) 2D map of the kurtosis of the dust temperature distribution along the line of sight, truncated at the $5 \sigma$ column density level.

\footnotetext{
${ }^{3}$ http://www.astro.cf.ac.uk/research/PPMAP_M31/
}

This paper has been typeset from a $\mathrm{T}_{\mathrm{E}} \mathrm{X} / \mathrm{L} \mathrm{T} \mathrm{E} \mathrm{X}$ file prepared by the author. 